\title{
El principio de objetividad en el ejercicio de la potestad administrativa sancionadora
}

\author{
Francisco José Rodríguez Pontón \\ Profesor titular de Derecho Administrativo. Universidad de Barcelona \\ francescrodriguez@ub.edu
}

\begin{abstract}
La aplicación del principio de objetividad al ejercicio de la potestad administrativa sancionadora requiere distinguir entre los diversos matices del significado de dicho principio así como entre la pluralidad y diversidad de situaciones que pueden dar lugar a la incoación de un procedimiento sancionador. En este trabajo se presta especial atención a las implicaciones de la objetividad en el ejercicio de la potestad sancionadora que pueden resultar especialmente relevantes ante la existencia de márgenes de apreciación en dicho ejercicio. Ya se trate de márgenes de apreciación en cuanto a la apreciación de los hechos, de la infracción tipificada, o a la determinación de la concreta sanción a aplicar, el principio de objetividad debe desplegar sus efectos de una manera que vaya más allá de una pura operación de constatación de hechos y de subsunción en una norma. La complejidad de ciertas situaciones y en ciertos entornos obliga en este sentido a destacar el valor de la adopción y el seguimiento de modo claro, motivado y coherente de unos criterios de decisión. Un mayor rigor en este proceder redundará sin duda en una mayor eficacia en la tutela de los intereses generales con el respeto debido a las garantías propias de un Estado de Derecho.
\end{abstract}

Resumen

Palabras clave Potestad administrativa sancionadora, Objetividad, Motivación, Margen de apreciación Apreciación de los hechos, Determinación de las sanciones, Adopción de criterios de decisión

\section{The principle of objectivity in the exercise of the administrative authority to impose sanctions}

\begin{abstract}
The application of the principle of objectivity to the exercise of the administrative authority to impose sanctions requires distinguishing the different nuances of the meaning of this principle and the plurality and diversity of situations that can give rise to the opening of a sanctioning procedure. This work particularly focuses on the implications of objectivity in the exercise of the authority to impose sanctions, which can be especially relevant in the light of the existence of margins of appreciation in this exercise. Whether the margins of appreciation are in the assessment of the facts or the offence defined in the law or in the establishment of the specific sanction to be imposed, the principle of objectivity should exert its effects in a way that goes beyond the mere exercise of corroborating the facts and categorising them into a law. In this respect, the complexity of particular situations in particular environments makes it important to stress the value of adopting and following certain decision-making criteria in a clear, reasoned and consistent manner. More rigour in this effort will no doubt result in greater effectiveness in the protection of the general interests with due respect for the guarantees of the rule of law.
\end{abstract}

Key words

Administrative authority to impose sanctions, Objectivity, Statement of reasons, Margin of appreciation, Assessment of the facts, Establishment of sanctions, Adoption of decision-making criteria 


\section{LOS SIGNIFICADOS DEL PRINCIPIO DE OBJETIVIDAD Y LA PERSPECTIVA DE SU APLICACIÓN CONCRETA AL ÁMBITO DEL EJERCICIO DE LA POTESTAD ADMINISTRATIVA SANCIONADORA}

El principio de objetividad caracteriza institucionalmente la Administración Pública desde el planteamiento constitucional del art. 103.1 de la Constitución española (CE), dado que, antes de realizarse una mención a otros principios (eficacia, jerarquía, descentralización, desconcentración y coordinación, con sometimiento pleno a la ley y al Derecho) se establece que la Administración Pública sirve con objetividad los intereses generales.

Este principio debe aplicarse a toda actuación administrativa. La actividad sancionadora no es una excepción. Sin embargo, es fácil constatar que en el momento de ser identificados los principios del ejercicio de la potestad administrativa sancionadora, el de objetividad no suele estar presente de manera explícita entre los mismos. Por no tomar más que un ejemplo, en la Ley 30/1992, de 26 de noviembre, de régimen jurídico de las Administraciones Públicas y del procedimiento administrativo común (LRJPAC) se establecen, por lo demás siguiendo de manera básica la jurisprudencia previa del Tribunal Constitucional, los principios de legalidad; irretroactividad, tipicidad, responsabilidad, proporcionalidad y los relativos a la prescripción y a la concurrencia de sanciones, por lo que se refiere a los principios de la potestad y siguiendo el orden que establece dicha ley.

Es obvio que ello no implica la inaplicación de dicho principio a las sanciones administrativas; ni dejar de ser conscientes de las evidentes conexiones del mismo con algunos de los indicados -como los de legalidad y proporcionalidad-; pero sí que parecería sugerir otra cosa, que no sería un principio especialmente característico del ejercicio de dicha potestad, o quizás que no requiere en general de especiales explicaciones, como sí las requeriría, por ejemplo, el principio de legalidad, tanto en más (por lo que se refiere a la especial intensidad de dicho principio cuando de sancionar se trata) como en menos (cuando se trata de establecer los casos que obligan a flexibilizar sus exigencias).

Esta observación iría estrechamente vinculada a ciertos presupuestos, o concepciones previas quizás no siempre explícitas, en relación con la manera de entender estos dos sujetos de la relación: el propio principio de objetividad y el ejercicio de la potestad sancionadora. En efecto, tanto en lo que se refiere a los contenidos concretos que traducen la exigencia de la objetividad como en lo que se refiere a las características que se predican del despliegue de la potestad sancionadora pueden concurrir una serie de elementos que quizás, como hipótesis, han dificultado, al menos en nuestra tradición jurídico-administrativa, un aprovechamiento de todo el potencial que puede aportar la aplicación del principio de objetividad al ejercicio de la potestad sancionadora. Veamos brevemente cada uno de estos dos elementos, de forma des-

212 doblada en el segundo de ellos. 


\subsection{Significados del principio de objetividad: actuación neutral; actuación con criterio}

No puede ser nuestro propósito aquí el de abordar toda la cuestión teórica de las distintas implicaciones o los distintos contenidos predicables del principio de objetividad, cuestión sobre la cual se pueden hallar aportaciones en este mismo volumen. Pero sí que creemos necesario realizar una observación general acerca de su percepción. Así, podemos decir que una primera presentación tendería a contemplar la objetividad desde la perspectiva de la imparcialidad o la neutralidad. De modo que se pone énfasis en el elemento de la ausencia de inclinación por determinados intereses, también de tipo político, o la necesidad de evitar interferencias y de actuar sin favoritismos ni discriminaciones, con neutralidad, con una eficacia indiferente. Éste es un aspecto que podemos encontrar como elemento característico en la explicación general del principio de objetividad. Y expresaría una tendencia a conectar directamente la objetividad del art. 103.1 CE con la imparcialidad del art. 103.3 CE, referida a la actividad de los funcionarios públicos'.

La diversidad de situaciones imaginables obliga en este sentido a considerar la presencia, como se ha dicho, de "supuestos de identificación y desidentificación entre objetividad e imparcialidad administrativa”" Sin embargo, más allá de este tipo de consideraciones, el principio de objetividad se ha presentado como una directiva de comportamiento de la Administración, particularmente relevante en el contexto del ejercicio de potestades discrecionales. En este caso, se pone énfasis en los elementos de la investigación de las circunstancias que concurren en cada caso y de la adecuada ponderación de los diversos intereses implicados. En este marco, se ha destacado un elemento de valor particularmente destacable, entendemos, en el contexto de las cuestiones aquí presentadas: la objetividad obliga a la Administración Pública a decidir en función del criterio que determine la normativa vigente; y a falta de éste, en función del criterio que aquélla determine como más adecuado para tomar la mejor decisión para los intereses generales³

$1 \quad$ Vid. las reflexiones críticas de Nieto García, Alejandro, “La Administración sirve con objetividad los intereses generales”, en Martín-Retortillo Baquer, Sebastián (coord.), Estudios sobre la Constitución española. Homenaje al profesor Eduardo García de Enterría, Civitas, Madrid, 1991, pp. 2228-2229. Puede verse también el manual de Sánchez Morón, Miguel, Derecho Administrativo. Parte general, Tecnos, Madrid, $7 .{ }^{\text {a }}$ ed., 2011, pp. 76-77.

2 Morell Ocaña, Luis, “El principio de objetividad en la actuación de la Administración Pública”, en Martín-Retortillo Baquer, Lorenzo (coord.), La protección jurídica del ciudadano (procedimiento administrativo y garantía jurisdiccional): estudios en homenaje al profesor Jesús González Pérez, Civitas, Madrid, 1993, pp. 152-153.

3 Sobre todos estos relevantes elementos pertenecientes a la concepción de la objetividad en este segundo sentido indicado vid. con detalle Ponce Solé, Juli, Deber de buena administración y derecho al procedimiento administrativo debido. Las bases constitucionales del procedimiento administrativo y del ejercicio de la discrecionalidad, Lex Nova, Valladolid, 2001, pp. 245-401. Santamaría Pastor destaca la doble implicación del principo de objetividad: fidelidad a los fines que marca la norma y necesidad de ponderación de los diversos intereses en presencia, siguiendo los criterios establecidos en la norma o criterios de congruencia y razonabilidad, generalmente admitidos y adecuados: Santamaría Pastor, Juan Alfonso, Princi-

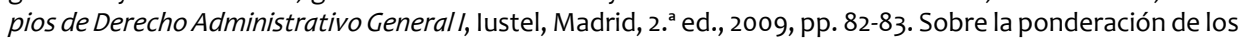


En cualquier caso, la objetividad, entendida como la obligación de actuar por parte del agente de la Administración sin aplicar otra voluntad que la de la ley, como ejecutor de la legalidad, no puede ignorar que en muchos casos la propia ley, o bien concede un margen en lo que se refiere a la operación de apreciar el supuesto de hecho, o bien permite la opción entre diferentes alternativas en cuanto a la consecuencia jurídica, lo cual no significa decidir libremente, sino conforme a una aplicación razonable de la ley ${ }^{4}$.

Intentaremos estar especialmente atentos a este elemento relativo al establecimiento y seguimiento de criterios de decisión en los espacios correspondientes a los márgenes de decisión existentes en el ejercicio de la potestad sancionadora.

\subsection{Ejercicio de la potestad sancionadora: aplicación de la ley; márgenes de apreciación}

El segundo de los elementos indicados sobre el cual se proyectan determinadas concepciones que pueden impedir un pleno aprovechamiento de la aplicación del principio de objetividad es el del ejercicio de la potestad sancionadora, y en particular en lo referente a su relación con la ley. Sin que ahora nos podamos centrar en el debate acerca del carácter discrecional o reglado del ejercicio de dicha potestad, es un hecho constatable que el sistema de garantías del que se ha dotado al sistema sancionador desde la Constitución de 1978, por contraste y marcando diferencias con los excesos propios del régimen anterior ${ }^{5}$, ha hecho recaer el peso en el factor de la limitación del poder de decisión en manos de la Administración. La construcción jurisprudencial de un único ius puniendi a través del cual se aplican, aunque con matices, los principios del Derecho Penal a las sanciones administrativas tendería así a poner más énfasis en la peculiaridad de esa actividad sancionadora que en los demás elementos que podría compartir con el ejercicio del resto de poderes de decisión en manos de las Administraciones Públicas.

La negación de la existencia de una verdadera discrecionalidad en el ejercicio de la potestad sancionadora formaría parte de este enfoque, como elemento de garantía que pasa necesariamente por la limitación del poder de decisión administrativa, que se entendería especialmente necesaria cuando de sancionar se trata. Una jurisprudencia bastante abundante ha ido estableciendo, en este sentido, que no resulta

\footnotetext{
diversos intereses en el marco de la definición y caracterización de los intereses generales, Nieto García, “La Administración sirve...", op. cit., pp. 2208-2209 y 2241-2244.

4 En este sentido vid. Morell Ocaña, “El principio de objetividad...”, op. cit., especialmente pp. $148-150$.

5 La presencia de elementos del régimen franquista ante los que había que dejar claras las garantías del Estado de Derecho queda puesta de manifiesto en algunas de las primeras sentencias del Tribunal Constitucional, como la 18/1981, de 8 de junio, en relación con las sanciones “de plano” en la legislación de
} 214 nal” (FJ 3). 
admisible la presencia de aspectos discrecionales en el ejercicio de la potestad sancionadora, discrecionalidad asociada a la idea de libertad de elección entre distintas soluciones jurídicamente indiferentes ${ }^{6}$. Diríase así que existe un importante elemento de sospecha en relación con la presencia de la discrecionalidad desde el punto de vista de la necesidad de respetar el sistema constitucional de garantías. Así, como se ha dicho en una de las monografías más recientes, "la discrecionalidad siempre se ha visto como algo opuesto a las sanciones"?.

Aunque es cierto que, junto a esta línea jurisprudencial, existe otra línea más matizada, sobre la que luego trataremos, parece clara la vinculación entre esta concepción limitadora y una determinada visión de la relación entre la ley y la aplicación de las sanciones. Una relación presidida por la idea de una legalidad concebida de forma rígida a la que debe corresponder una tarea de la Administración a la hora de sancionar básicamente de subsunción de hechos y de automatismo en la aplicación de las consecuencias. En palabras difíciles de superar a la hora de ilustrar la situación, se ha dicho que

“[...] se concibe el Derecho Administrativo Sancionador como un ordenamiento heterónomo a la Administración y a los tribunales [...]. [...] en la actividad aplicativa de las normas se asigna a los funcionarios y jueces una tarea esencialmente automática ya que tienen que limitarse a realizar continuas operaciones formales de subsunción, o sea, encajar unos hechos "objetivos" determinados por el principio de la presunción de inocencia, en unos tipos legales rigurosamente preestablecidos en la ley. Fuera del círculo iluminado por la ley, no hay más que las tinieblas de lo ilícito, de lo prohibido"s.

Nos interesa remarcar al hilo de estas consideraciones dos elementos vinculados a las mismas.

En primer lugar, es posible detectar una concepción de lo objetivo que toma únicamente en cuenta la constancia de unas conductas en una norma, conductas que luego deben ser simplemente detectadas a partir de descripciones en las que cabe subsumir los hechos de la realidad. Podríamos hablar así de una concepción pasiva de la objetividad, frente a una de tipo activa que aquí vamos a intentar subrayar.

En segundo lugar, cabe destacar el peso de una concepción negativa por esencia de los espacios que quedan por determinar al margen de la estricta definición legal, espacios que deberían así tender a ser limitados.

6 Así, por ejemplo, en sentencias como la Sentencia del Tribunal Supremo (STS) de 23 enero 1989 (Ar. 421, FJ 1) y otras que reproducen de modo muy similar sus razonamientos.

$7 \quad$ Huergo Lora, Alejandro, Las sanciones administrativas, lustel, Madrid, 2007, p. 247.

8 Nieto García, Alejandro, Derecho Administrativo Sancionador, Tecnos, Madrid, 4. ${ }^{a}$ ed., 2005, p. 249 . 


\subsection{Sanciones: determinación de consecuencias jurídicas; apreciación de concurrencia de hechos}

La caracterización de las concepciones de mayor peso del ejercicio de la potestad sancionadora quedaría incompleta sin referirnos al aspecto relativo al lugar ocupado por los hechos y por las consecuencias jurídicas. La objetividad y la legalidad de la actuación sancionadora vendrían aseguradas por la operación de subsunción de unos hechos en unos tipos definidos por la norma, las infracciones, de cuya concurrencia se extraen las debidas consecuencias jurídicas, las sanciones. Cualquier margen de decisión relevante en este proceso, especialmente en su primera fase, tendería a ser visto poco menos que como una veleidad contraria a las garantías constitucionales.

Corre paralela, esta concepción, a una determinada forma de entender la manera de ser determinados los hechos en el mundo del Derecho, y en consecuencia a su peso específico frente a la operación de la determinación de las consecuencias jurídicas, y ya no sólo en el ámbito de las sanciones, sino con carácter más general. Frente a una concepción más tradicional que tiende a presentar los hechos como elementos constatables y no sometidos a especiales procesos de construcción o de apreciación, creemos que cabe remarcar una línea que ya se ha expuesto por ejemplo en relación con los ámbitos en los que actúan las autoridades independientes. Línea que pasa por señalar, de acuerdo con las enseñanzas de la epistemología contemporánea, el carácter cognitivo y racional de la operación de fijación de los hechos, proceso opinable, refutable y controlable y que además presupone un margen de apreciación en manos de la autoridad que toma decisiones y en la que resulta clave la motivación a través de la fijación de criterios que guiarán las posteriores decisiones ${ }^{9}$. Y es que, frente a la aparente sencillez de la caracterización de una conducta como objetiva, se alza la dificultad práctica de su concreción teniendo en cuenta que "con frecuencia los hechos no son nada claros, y mucho menos obvios, antes al contrario discutibles y sujetos indefectiblemente a valoraciones subjetivas"10.

Destacar los márgenes de decisión existentes, no sólo en la determinación de la consecuencia jurídica -por ejemplo en la graduación de la sanción- sino también en la determinación de la concurrencia misma de los hechos, es algo que puede resultar especialmente interesante para una aplicación completa de las facetas presentes en el principio de objetividad, además de necesario ante la creciente complejidad de muchos sectores de la realidad en los que la apreciación de los hechos dista de ser obvia.

9 Ideas planteadas y desarrolladas por Malaret i García, Elisenda, "Autoridades independientes y justicia administrativa”, en Montoro Chiner, María Jesús (coord.), La Justicia Administrativa. Libro homenaje al Prof. Dr. D. Rafael Entrena Cuesta, Atelier, Barcelona, 2003, especialmente pp. 762-767.

10 Nieto García, “La Administración sirve...”, op. cit., pp. 2227-2228. 


\subsection{La necesidad de contemplar la diversidad de las facetas que traducen el principio de objetividad en el ejercicio de la potestad sancionadora}

La propia diversidad de significados que puede albergar la noción de objetividad hace posible su presencia diversificada en la actuación administrativa cuando de ejercer la potestad sancionadora se trata. Vamos a tratar de exponer, básicamente a través de un estudio jurisprudencial, con cierto orden, las dimensiones o facetas más destacables de esta presencia, en los aspectos que nos parecen más relevantes teniendo en cuenta las evoluciones actuales de los contextos en los que se desarrolla el ejercicio de dicha potestad. Concretamente centraremos nuestra atención, por ser un aspecto que creemos especialmente necesario subrayar, en la faceta vinculada con la necesidad de concretar márgenes de apreciación, en que la referencia a la necesidad de adoptar y seguir criterios objetivos se muestra especialmente necesaria.

Dejamos fuera de este estudio, pues, otras cuestiones conectadas con el principio de objetividad en el ejercicio de la potestad sancionadora, como las relativas a las garantías de imparcialidad y objetividad de los órganos que intervienen en el procedimiento sancionador o las relativas a la separación entre las fases de instrucción y resolución, que son otra muestra de la presencia polifacética de este principio.

La necesidad de contemplar la presencia del principio de objetividad a través de una diversidad o de una escala de intensidad plasmada en facetas distintas deriva a su vez, además del aspecto vinculado a sus diversos matices de significado, de la propia diversidad que albergan los posibles supuestos de infracciones y sanciones administrativas. En efecto, la enorme diversidad presente de casos, el muy variado espectro posible de grados de complejidad de su apreciación y de su determinación, hace que el grado de elaboración de la presencia del principio de objetividad también sea distinto. En aquellos casos que se presenten de manera más sencilla, con criterios más predeterminados o con escasos márgenes de apreciación, la objetividad puede venir identificada con su sentido más básico relativo a la vinculación de la decisión a la norma jurídica, traducida en una motivación que acredite la concurrencia de unos hechos definidos en la misma y que identifique la norma jurídica aplicable. Por el contrario, como mencionábamos, supuestos de mayor complejidad, en cuanto a los hechos, en cuanto a las posibles consecuencias o en cuanto a las circunstancias concurrentes, requerirán una mayor profundización de los contenidos del principio de objetividad, que requerirá también de una mayor elaboración.

\section{OBJETIVIDAD, APRECIACIÓN DE HECHOS Y DETERMINACIÓN DE SANCIONES. ESPECIAL ATENCIÓN AL ELEMENTO DE LA ADOPCIÓN Y SEGUIMIENTO DE CRITERIOS DE DECISIÓN}

\subsection{Objetividad, valor probatorio y determinación de hechos}

Una de las manifestaciones más básicas de la presencia del elemento vinculado a la objetividad en la actuación sancionadora de la Administración por parte de la jurisprudencia viene dada por la atribución de valor probatorio a las actas de inspección. En este 
caso, se trata de acotar el reconocimiento de su presunción de veracidad, que el art. 137.3 LRJPAC establece con carácter general, señalándose que dicha presunción se limita "a sólo los hechos que por su objetividad son susceptibles de percepción directa por el inspector o a los inmediatamente deducibles de aquéllos o acreditados por medios de prueba consignados en la propia acta". Se trata de destacar que el valor probatorio de estos documentos va ligado a la "percepción directa" del funcionario y a la "circunstanciación detallada" de los hechos. Rigor, pues, en la consignación de los hechos y en la determinación de su fuente de adquisición, que iría vinculado a la objetividad a los efectos de atribución del valor probatorio ${ }^{11}$. Y que en algún caso ha ido acompañado de la observación acerca de la dificultad práctica de aplicación de la apreciación en casos como aquellos en que se debe producir un "riesgo grave e inminente" para la salud o la integridad de los trabajadores, dificultad que obliga a valorar todas las circunstancias concurrentes ${ }^{12}$.

En alguna ocasión la jurisprudencia establece la "presunción de objetividad e imparcialidad" de determinados análisis efectuados por centros oficiales, conectada a la falta del "menor interés concreto y personal" de quienes los llevan a cabo, destacándose además su carácter “concienzudo y detallado". En este caso, relativo a una intoxicación en restaurante, además destaca cómo se hace también alusión a la intervención de las "reglas del criterio humano" en la prueba de presunción"3.

También hallamos algún caso relativo a infracciones en materia de prevención y extinción de incendios forestales en cuanto, eso sí, a la fijación del importe de los daños y perjuicios ocasionados, en los que se valora la objetividad de la prueba, a propósito de informes periciales de los que se destaca su carácter "fundado, ponderado y racional", así como sus virtudes comparadas con otros informes ${ }^{14}$. Referencias también a la idoneidad y objetividad de determinados tipos de prueba, que, a la vista de las condiciones de su práctica, son importantes en casos como los relativos a infracciones en materia de elaboración de ciertos alimentos ${ }^{15}$.

Valga esta breve referencia para tener en cuenta la presencia de la objetividad en el mundo de los hechos, de su apreciación y constatación.

\subsection{Conceptos jurídicos indeterminados, márgenes de apreciación y establecimiento de criterios}

Dando un paso más, de la percepción de los hechos a su encaje en las normas, es bien conocido, y así ha sido expuesto por la doctrina ${ }^{16}$, cómo la jurisprudencia, y de

11 Podemos destacar en esta línea, entre otras, la STS 23 marzo 1998 (Ar. 2862), FFJJ 3 y 4. Así como la STS 22 abril 1991 (Ar. 3313, FJ 2), donde se opone la "aportación de pruebas objetivas" a un informe que supone "la expresión del juicio" del inspector.

12 STS 27 febrero 1998 (Ar. 2543, FJ 3).

13 STS 9 febrero 1988 (Ar. 804, FFJJ 5 y 6$)$.

14 STS 25 junio 2010 (Ar. 5898, FFJJ 2 y 4).

15 Así, en el caso de la STS 16 junio 1982 (Ar. 4812, FJ 2).

16 Vid., por ejemplo, Lasagabaster Herrarte, Iñaki, “Artículo 4. Tipicidad”, en Lasagabaster Herrar-

218 te, Iñaki (dir.), Ley de la potestad sancionadora. Comentario sistemático, LETE, IVAP, Bilbao - Iruña, 2006, 
modo destacable en este caso la del Tribunal Constitucional, se ha pronunciado sobre la posibilidad de la presencia de conceptos jurídicos indeterminados en la tipificación de infracciones administrativas. Si destacamos este punto es porque entendemos que ello resulta una premisa importante para un margen de aplicación del principio de objetividad en su faceta más activa, es decir, la consistente en la necesidad de contar con criterios objetivos para la correcta aplicación de dichos conceptos.

En efecto, el Tribunal Constitucional ha establecido, en el ámbito penal, que el principio de legalidad es compatible con el hecho de que en la definición de ciertos tipos se utilicen conceptos para cuya delimitación se "permita un margen de apreciación", sobre todo tratándose de la protección de bienes jurídicos reconocidos internacionalmente y en casos en que su concreción es "dinámica y evolutiva". Así se estableció en la Sentencia del Tribunal Constitucional (STC) 62/1982, de 15 de octubre (FJ 7).

En dos conocidas sentencias posteriores del año 1989, el Tribunal Constitucional ya se refirió al ámbito administrativo sancionador. Señaló entonces que no es contraria a la exigencia de lex certa la regulación de ilícitos mediante conceptos jurídicos indeterminados, "siempre que su concreción sea razonablemente factible en virtud de criterios lógicos, técnicos o de experiencia y permitan prever con suficiente seguridad, la naturaleza y las características esenciales de las conductas". Afirmación contenida en la STC 69/1989, de 20 de abril (FJ 1) y en la STC 219/1989, de 21 de diciembre (FJ 5). La primera de las cuales señalaba que es imposible que los conceptos legales alcancen "una claridad y precisión absolutas", factor que hace necesario ese margen de indeterminación. La presencia de los criterios interpretativos señalados salvaría la seguridad jurídica necesaria y el principio de legalidad, aspecto que cabe remarcar. Criterios en los que será relevante la actuación coherente y motivada de los aplicadores y en los que el principio de objetividad deberá tener un importante papel.

La jurisprudencia del Tribunal Supremo, en diversos contextos y con diversos matices, ha partido de estas premisas. Podemos destacar algunas referencias concretas.

Así, en un caso relativo a imputación de retraso en el desempeño de la función judicial, la STS 3 junio 1994 (Ar. 7374, FFJJ 2 y 4) establece que tal concepto, como motivo determinante de una infracción disciplinaria, es un concepto indeterminado que exige para concretarlo la utilización de distintos criterios conjuntamente, que se detallan, teniendo en cuenta circunstancias anómalas que puedan estar presentes así como apreciaciones tanto cuantitativas como cualitativas.

pp. 124-134; y Pemán Gavín, Ignacio, El sistema sancionador español. Hacia una teoría general de las infracciones y sanciones administrativas, Cedecs, Barcelona, 2000, pp. 229-243. Sobre el grado de precisión exigible a las normas penales desde la perspectiva de la jurisprudencia del Tribunal Europeo de Derechos Humanos y la consideración de la importancia en esta cuestión de los contextos evolutivos, puede verse Scoletta, Marco, “El principio de legalidad penal europeo”, en Díez-Picazo, Luis María y Nieto Martín, Adán (dirs.), Los derechos fundamentales en el Derecho Penal Europeo, Civitas-Thomson, Cizur Menor, 2010, pp. $269-270$. 
Existen unos casos en los que la referencia a la indicada jurisprudencia del Tribunal Constitucional constituye la base de votos particulares contrarios al criterio mayoritario, en el ámbito de la legislación laboral, criterio consistente en considerar que la tipificación contenida en la normativa aplicable ${ }^{17}$ no permite "objetivar apriorísticamente la correspondencia precisa entre la sanción y la infracción”, permitiendo al órgano sancionador "actuar con excesivo arbitrio", "y no con el prudente y razonable que permitiría una debida especificación normativa". Es el caso de las SSTS 28 septiembre 1990 (Ar. 6865, FFJJ 3 del voto particular y 4 de la sentencia) y de 5 octubre 1990 (Ar. 8006, FFJJ 3 del voto particular y 2 de la sentencia).

En otros casos, se destaca la suficiencia de la tipificación de ciertas conductas. Por ejemplo, en el ámbito disciplinario, en que, en la STS 15 diciembre 2000 (Ar. 9853, FJ 5) se indica que conductas como las imputadas son "perfectamente previsibles" para cualquier otro sujeto (en aquel caso estudiante) "normal”, siendo de "manifiesta previsibilidad" la conducta sancionable y su gravedad teniendo en cuenta las obligaciones que asumen las personas que entran en relaciones de sujeción especial. En el caso de la STS 1 octubre 1992 (Ar. 7750, FJ 4), en materia de la regulación del suministro y venta de gasolinas y gasóleos de automoción, simplemente se decide que, frente a las alegaciones de incompatibilidad con el principio de legalidad por uso de conceptos jurídicos indeterminados, conceptos como "antelación precisa" en la formulación de pedidos o "insuficiente atención” de los aparatos surtidores, en el marco de las descripciones en las que se sitúan, "reflejan supuestos de hecho perfectamente identificables o susceptibles del adecuado control en su aplicación”.

Quizás una mención particular merecen casos como el de la STS 17 mayo 2007 (Ar. 5857, FJ 2), en que, tratándose de unos actos sin carácter sancionador, dentro de las "medidas necesarias para salvaguardar la libre competencia en el mercado" por parte de la Comisión del Mercado de las Telecomunicaciones, se establece que pese a la indeterminación de dicho concepto de "medidas necesarias", no cualquiera puede ser válida, sino que deben ser proporcionadas y suficientemente motivadas, dado que su incumplimiento podría originar una conducta tipificada como infracción administrativa, siendo exigible su previa concreción.

Aunque la diversidad de casos y también de enfoques y del nivel de las soluciones adoptadas hace muy difícil establecer una conclusión uniforme, sí que creemos que la jurisprudencia permite captar un hecho esencial. La imposibilidad de que la misma predetermine todas las conductas imaginables obliga a dotar de una cierta indeterminación el enunciado de las conductas sancionables, lo cual a su vez obliga a determinar criterios para su apreciación, criterios que el juez puede controlar; pero sobre la base de una tarea en la que a la Administración corresponde un inevitable rol de determinación de pautas en las que debe moverse respetando el principio de objetividad; pero como algo que va más allá de la mera constatación de hechos conteni-

17 Casos referidos al derogado art. 57 del Estatuto de los Trabajadores que el Tribunal Constitucional también censura en las sentencias 207/1990, de 17 de diciembre y 40/1991, de 25 de febrero. 
dos en las normas. Todo ello puede adquirir mayor importancia en el contexto de ciertos ámbitos, como los supervisados por autoridades independientes, donde es frecuente atribuir poderes de intervención a partir de conceptos indeterminados que conforman el hecho determinante, como "mercado relevante", "estabilidad del mercado financiero", "posición dominante”, etc $^{18}$.

En estos o en otros casos, aunque no se reconozca la presencia de una verdadera discrecionalidad, lo que parece indudable es que está presente un margen de apreciación, en cuyo ejercicio la Administración debe ponderar factores y debe adoptar criterios objetivos de actuación. Y es que, ya se trate de auténtica discrecionalidad o de presencia de conceptos indeterminados, como bien se ha indicado, las cosas cambian poco de cara a la necesidad de motivación, ya que se trata de operaciones de interpretación que requieren toma de decisiones, por no tratarse de meras deducciones ${ }^{19}$, y cuyos criterios por tanto se han de explicar.

\subsection{La motivación y la adopción de criterios de decisión}

La ley establece de modo explícito, más allá del contenido general del art. 54 LRJPAC, el deber de motivación de "la resolución que ponga fin al procedimiento [sancionador]", debiendo resolver ésta "todas las cuestiones planteadas en el expediente" (art. 138.1 LRJPAC).

La obligación de motivar las decisiones sancionadoras adquiriría, visto a través del prisma del establecimiento y seguimiento de unos determinados criterios en el marco de los espacios de apreciación, un sentido más denso que iría más allá de la mera indicación de la norma aplicada a los hechos concretos, propia de la operación de subsunción, justificando así la conducta en la que se encuadra la acción sancionada y la determinación precisa de la sanción adoptada. Siendo ello por supuesto necesario, la motivación de las sanciones debería tener mayor alcance ${ }^{20}$ e incluiría una referencia a los criterios adoptados en la tarea de concretar la presencia de los conceptos indeterminados cuya existencia da pie a la apreciación de los hechos. La STS 20 junio 2006 (Ar. 4634, FJ 5) verifica el cumplimiento de las exigencias de motivación de las decisiones judiciales,

18 Pone de relieve la importancia de estos procesos en estos casos Malaret i Garcia, “Autoridades independientes...", op. cit., pp. 760-762.

19 Igartua Salaverría, Juan, La motivación en los nombramientos discrecionales, Thomson-Civitas, Cizur Menor, 2007, p. 192. Vid. también sobre la necesidad de actos de valoración que implican los conceptos indeterminados y sobre los argumentos que relativizan sus diferencias con la discrecionalidad, Nieto García, “La Administración sirve...”, op. cit., pp. 2246-2249.

20 Vid. una completa explicación de los elementos que deben aparecer en la motivación de la resolución sancionadora, incluyendo las razones o valoraciones consideradas para la imposición de una sanción concreta; los criterios de graduación aplicados, el modo concreto de su aplicación, etc., en Izquierdo Carrasco, Manuel, “La resolución sancionadora”, en Rebollo Puig, Manuel; Izquierdo Carrasco, Manuel; Alarcón Sotomayor, Lucía y Bueno Armijo, Antonio M. a, Derecho Administrativo Sancionador, Lex Nova, Valladolid, 2010, pp. 765-781. 


\begin{abstract}
"que se integra en el derecho a la tutela judicial efectiva que garantiza el art. $24 \mathrm{CE}$, y que comporta en primer término el reconocimiento del derecho a obtener una resolución fundada en Derecho favorable o adversa, porque contiene los elementos y razones de juicio que permiten conocer cuáles han sido los criterios jurídicos seleccionados para fundamentar su decisión".
\end{abstract}

A su vez, cuando se trata de motivar una resolución judicial que concuerda con una decisión administrativa objeto de impugnación, se debe poner la atención en cómo la sentencia razona, y si lo hace de modo claro y exhaustivo, sobre las causas que hacen que estime "justificado, razonable, ausente de arbitrariedad y motivado el criterio de la administración", como se establece en el caso de la STS 29 enero 2008 (Ar. 929, FJ 4), referido al criterio relativo a la aminoración del impacto visual y estético de una obra en un entorno protegido.

Esa tarea de "selección" o de determinación de los criterios que sirven para decidir en los espacios correspondientes a los márgenes de apreciación y esa tarea de examinar la justificación de los criterios adoptados, sería también fundamental en la motivación de las decisiones administrativas.

Este rigor necesario en la toma de decisiones pasará a menudo por la formulación o adopción misma de los criterios para decidir, previamente a su aplicación concreta. Como se ha dicho, "la imparcialidad comienza ya con la adopción de los criterios de actuación" "21. Lo cual, siendo especialmente importante en el ámbito de las decisiones "técnico-discrecionales", especialmente en el ámbito de las comisiones que resuelven concursos y oposiciones, para el principio de imparcialidad, lo puede ser igualmente en general para el principio de objetividad, con el que está emparentado. Hay que destacar el peso específico de este elemento, que es el que traduce las exigencias de una motivación suficiente. En este sentido, creemos de suma importancia el elemento que ha sido destacado, a la vista de cierta jurisprudencia, acerca del significado de esta suficiencia de la motivación: la motivación que basta para conocer la fundamentación que respalda la decisión, para poderla combatir adecuadamente; la que permite conocer "cuáles son los criterios a aplicar, cómo se han aplicado y, finalmente, si se aplican por igual a todos los concernidos"22.

Esta necesidad de establecer criterios generales a partir de los cuales tomar decisiones concretas ha sido explicitado de modo particularmente claro en algún ámbito, como el de la determinación de las indemnizaciones por daños y perjuicios ocasionados al dominio público hidráulico, donde la jurisprudencia ha hecho hincapié en el requerimiento normativo sectorial de fijación de criterios generales al respecto. De modo que no es posible dar por buena una valoración de tales daños contenida en una resolución administrativa sin verificar si se acomoda a unos criterios generales

21 Igartua Salaverría, Juan, Discrecionalidad técnica, motivación y control jurisdiccional, Civitas, Madrid, 1998, p. 134.

22 Igartua Salaverría, Discrecionalidad técnica..., op. cit., p. 164. 
previamente establecidos, como dice la STS 10 diciembre 2010 (Ar. 8949, FJ 3). Lo cual además, aunque se trate de la determinación de unos daños y perjuicios, incide directamente en el ejercicio de la potestad sancionadora, ya que su tipificación en uno u otro grado presupone la causación de determinados daños, que deben ser, pues, cuantificados, como se observa en el caso de la STS 1 febrero 2010 (Ar. 1368, FJ 5).

Todo ello también puede y debe ser así cuando se trata de la apreciación de hechos sancionables. Y es que a mayor presencia de conceptos indeterminados correspondería una exigencia más precisa de motivación de la resolución sancionadora ${ }^{23}$.

No cabe olvidar que, al igual que en otros tipos de decisiones, la apreciación de los hechos que pueden desencadenar un procedimiento sancionador se puede producir en un entorno de notable complejidad que justifica la atribución de un margen de apreciación, contrarrestado por una exigente obligación de motivación. Así sucede por ejemplo con la actuación de la Comisión de la Unión Europea en casos de infracciones en materia de competencia. Casos en los que se afirma que el control jurisdiccional de las decisiones de la Comisión "sobre valoraciones económicas complejas" "se limita" a comprobar el respeto de las normas de procedimiento y de motivación, la exactitud material de los hechos y la falta de error manifiesto de apreciación y de desviación de poder ${ }^{24}$.

En sectores como el de la supervisión del mercado de valores se ha señalado el carácter central del deber de motivar sus decisiones por parte de las autoridades, siendo así que, si se trata de garantizar la transparencia del mercado financiero, nada sería más incoherente que no asegurar una claridad en sus procesos decisorios; factor que puede resultar de especial importancia para evitar la arbitrariedad en la imposición de sanciones ${ }^{25}$.

Evidentemente no todas las situaciones son iguales. De ahí también que el deber de motivación variará, y con ello también el énfasis puesto en el elemento vinculado al establecimiento de criterios, en función de la complejidad de cada caso. Como se ha dicho, "cuanto mayor sea la variedad y la complejidad de las valoraciones que efectúa el órgano administrativo, más completa y compleja habrá de ser la motivación de lo que aquél ha decidido"26.

En materia sancionadora, la motivación en muchos casos puede ser suficiente con la referencia a los hechos constatados y la normativa aplicable. Y la objetividad en este plano consistiría en proceder de manera fiel a los textos legales, sin interferen-

\footnotetext{
23 Vid. Lasagabaster Herrarte, “Artículo 4...”, op. cit., p. 129.

24 Por ejemplo en la Sentencia del Tribunal de Justicia de la Unión Europea (STJUE) de 14 octubre 2010 (asunto C-280/08 P), apartado 143.

25 Vid. a este respecto las interesantes observaciones críticas de Simon, François-Luc, Le juge et les autorités du marché boursier, LGDJ, París, 2004, pp. 207-228.

26 Igartua Salaverría, Discrecionalidad técnica..., op. cit., p. 135, citando también en este mismo sentido a M. Sánchez Morón.
} 
cias indebidas. En otros, puede ser necesario un mayor trabajo de concreción tanto en la determinación de los hechos como de las concretas sanciones. En estos casos la objetividad abarcaría esta faceta más rica, compleja y activa de la adopción y seguimiento de criterios. La gama de situaciones intermedias es sin duda extensa, como hemos puesto de relieve.

\subsection{Determinación concreta de la sanción correspondiente, márgenes de apreciación, objetividad y motivación}

La determinación concreta de la sanción que debe corresponder a las conductas que constituyen infracción ${ }^{27}$ acostumbra a ser un espacio de un cierto mayor reconocimiento de márgenes de apreciación en manos de las Administraciones Públicas y por tanto generador quizás de mayores expectativas de aplicación del principio de objetividad en el sentido activo que aquí tratamos de priorizar.

Por enlazar con el caso del Derecho de la Unión Europea hace un momento comentado, es claro el reconocimiento por parte de la jurisprudencia comunitaria de que la Comisión dispone de un margen de apreciación para determinar los importes de las multas en materia de defensa de la competencia, como se reconoce por ejemplo en la STJUE 29 septiembre 2011 (asunto C-520/09 P) (apartado 93). Muy clara en este sentido es la Sentencia del Tribunal General de 25 octubre 2011 (asunto T-348/08, apartado 293), dado que no sólo recuerda que es jurisprudencia reiterada que la Comisión dispone de dicho margen de apreciación, sino que destaca la finalidad de dicha atribución, "de lograr que las empresas ajusten su comportamiento a las normas sobre competencia"; razonando que "la aplicación eficaz de las normas comunitarias sobre competencia exige [...] que la Comisión pueda en todo momento adaptar el nivel de las multas a las necesidades de esta política [la política comunitaria sobre la competencia]".

En este aspecto hay matices que deben ser introducidos también en lo referente a la jurisprudencia española, que en este punto no ha seguido siempre el dogma del carácter reglado de la imposición de las sanciones administrativas. Conviene detenerse un momento en ello.

En efecto, varios pronunciamientos jurisprudenciales han venido sosteniendo desde hace años que a la hora de fijar la cuantía de la multa no se le puede negar a la Administración una facultad para aplicar discrecionalmente, dentro de los límites legales, la sanción que resulte adecuada a las circunstancias del caso ${ }^{28}$. Eso sí, encontramos menciones también a la necesidad de motivar como contrapartida. No

27 Sobre los diversos aspectos implicados en esta operación vid. el completo artículo de Izquierdo Carrasco, Manuel, “La determinación de la sanción administrativa”, en Justicia Administrativa, número extraordinario, 2001.

28 Por ejemplo, STS 21 febrero 1979 (Ar. 680, FJ 3). 
por casualidad, otra vez en el ámbito de la defensa de la competencia se ha establecido que

\begin{abstract}
"Es claro que la decisión de la Administración en que se imponga la sanción ha de ser motivada; en otras palabras, la Administración ha de justificar, de manera objetiva, el porqué de la sanción que impone. Aún cabe añadir; la Administración, necesariamente, ha de acomodar su potestad sancionadora a cada caso. Por lo tanto, a la hora de imponer la sanción tras el correspondiente expediente, ha de tomar en consideración las circunstancias particulares de cada caso, así como -y esto es relevante- las circunstancias del mercado y producción dominantes al momento en que la infracción se cometió”29.
\end{abstract}

No parece demasiado aventurado pensar que esa justificación objetiva a la que se alude pasará por identificar criterios coherentes y previsibles de actuación.

De modo que, una cosa sería que los casos de falta manifiesta de concreción de determinados preceptos provoquen una remisión problemática a apreciaciones subjetivas concretas de la autoridad ${ }^{30}$, y otra que se reconozcan márgenes de apreciación o incluso de discrecionalidad en la determinación exacta de la sanción aplicable a cada caso, respetando el principio de proporcionalidad. La correspondencia entre infracciones y sanciones, ha dicho la STC 207/1990, de 17 de diciembre (FJ 3) "puede dejar márgenes más o menos amplios a la discrecionalidad judicial o administrativa, pero que en modo alguno puede quedar encomendada por entero a ella"31.

La jurisprudencia del Tribunal Constitucional se ha enfrentado a diversos casos de este tipo, y ha tenido que resolver acerca de la suficiencia de los contenidos de las normas a la hora de remitir a la apreciación administrativa la determinación exacta de las sanciones correspondientes. Una línea de respuesta que ha proporcionado el alto tribunal ha sido la de encontrar criterios legales aplicables a los que resultarían ser aparentes márgenes de decisión en manos de la Administración. Es decir, ante la aparente concesión de un espacio libre de decisión acerca de la posibilidad de imponer un determinado tipo de sanción en un precepto legal, se establece que le resultan de aplicación criterios "perfectamente definidos y objetivados" pertenecientes a otro precepto que regula los criterios de graduación de las sanciones. Solución establecida por la STC 14/1998, de 22 de enero, FJ 10, en materia de caza.

29 STS 22 octubre 1997 (Ar. 7542, FJ 7).

30 En este sentido, STS 19 abril 1983 (Ar. 2817, FJ 4).

31 En este contexto, el problema de la falta de determinación legal suficiente se puede contemplar como un caso en que el déficit de programación legal puede provocar una vulneración de la tutela judicial efectiva, dadas las limitaciones de fiscalización judicial de las sanciones que implicaría: vid. el análisis en este sentido de Bacigalupo, Mariano, La discrecionalidad administrativa (estructura normativa, control judicial y límites constitucionales de su atribución), Marcial Pons, Madrid, 1997, pp. 250-253. 
La misma senda sigue una resolución posterior, la STC 113/2002, de 9 de mayo, FFJJ 5-7, en materia de tráfico y seguridad vial. Sin embargo, esta sentencia contiene algunas afirmaciones que nos interesa destacar. Así, en primer lugar, se dice que

“[... ] debemos resaltar, ante todo, que la necesidad de que la ley predetermine suficientemente las infracciones y las sanciones, así como la correspondencia entre unas y otras, no implica un automatismo tal que suponga la exclusión de todo poder de apreciación por parte de los órganos administrativos a la hora de imponer una sanción concreta".

Referencia a la no exigibilidad de automatismo como corolario del principio de legalidad sancionadora que cabe destacar, dados ciertos elementos rígidos y formales presentes en el entendimiento de este aspecto que destacábamos al principio de este texto. Posteriormente la sentencia mencionada hace una referencia comparativa al Código Penal, aseverando que

“[... ] la atribución al órgano sancionador de una facultad discrecional de apreciación para decidir la imposición de tales penas o medidas no es algo extraño en el ordenamiento punitivo español, y cuenta con antecedentes precisos en la esfera penal, cuyas garantías son trasladables -con algunos matices- al ámbito sancionador administrativo".

Pese a que, como se ha visto, existe una tendencia a buscar la seguridad de los criterios en la propia ley, la actuación administrativa siempre deberá consistir, sobre todo en los casos de mayor complejidad, en una individualización de la sanción a la vista de una serie de circunstancias concretas. La STC 116/2007, de 21 de mayo, en un caso de sanción disciplinaria impuesta por el Consejo General del Poder Judicial, aprecia que dicha sanción no es desproporcionada, y ello porque, al verificar la motivación de la misma,

\footnotetext{
"se pone de manifiesto no sólo que se tuvieron en cuenta las concretas circunstancias concurrentes en el caso para efectuar la individualización de la sanción, sino que, además, fueron debidamente ponderadas en relación con los derechos de la recurrente que pudieran verse afectados" (FJ 7).
}

Consideración de las circunstancias y ponderación de los intereses que, como vimos, forma parte de los contenidos más plenos del principio de objetividad y que muy a menudo deberán ir acompañadas de la adopción y seguimiento de criterios de decisión, explicitados en mayor o menor medida, aspecto que será muy relevante en la determinación y graduación de las sanciones concretas a imponer.

\subsection{Algunas referencias concretas de interés a la necesidad de establecer criterios objetivos en el ejercicio de la potestad sancionadora}

La evolución de ámbitos donde la complejidad de las circunstancias es notable, 226 obligando a delicados ejercicios de valoración de las mismas también cuando de san- 
cionar conductas se trata se ha puesto de manifiesto de manera particular en ámbitos económicos. Vamos a destacar brevemente dos, el relativo a la defensa de la competencia y el relativo al mercado de valores.

Especial consideración merece, en primer lugar, el caso de la defensa de la competencia. Ya hemos visto aparecer este ámbito anteriormente, y sobre él se ha dicho, cabe recordar, que es un sector en el que "paradigmáticamente" los supuestos de hecho de los tipos de infracciones necesitan recurrir a conceptos indeterminados, ya que "sólo a título enumerativo puede establecerse qué conductas son susceptibles de ser sancionadas, pues, el bien jurídico protegido, la competencia efectiva, no permite mayores concreciones" 32 .

Pues bien, haremos ahora mención a una perspectiva reciente en la actuación de la autoridad independiente en este ámbito, la Comisión Nacional de la Competencia (CNC), que resulta muy destacable, tanto por el tipo de instrumento utilizado como por su contenido. Se trata de la posibilidad que tiene esta autoridad de publicar comunicaciones aclarando los principios que guían su actuación en aplicación de la Ley de Defensa de la Competencia de 2007 (LDC). Posibilidad que se ha traducido ya en la práctica en documentos de sumo interés para nuestro objeto de estudio.

Por un lado, conviene destacar especialmente la Comunicación sobre la cuantificación de las sanciones derivadas de infracciones de los artículos 1, 2 y 3 de la Ley 15/2007, de 3 de julio, de defensa de la competencia y de los artículos 81 y 82 del Tratado de la Comunidad Europea, de 6 de febrero de 2009, publicada en la página web de la Comisión Nacional de la Competencia. Aspecto que se incardinaría, pues, en el punto anteriormente visto, pero que exponemos aquí dada la peculiaridad de este mecanismo institucional. Sobre esta comunicación no vamos a entrar en aspectos de fondo; en cuál es la definición y los criterios de valoración concretamente adoptados; pero sí conviene destacar algunos elementos generales.

Para empezar, el texto comienza destacando, en su primer párrafo, la competencia sancionadora de la CNC, en cuyo ejercicio "dispone de cierto margen de apreciación en aplicación del principio de proporcionalidad, dentro de los límites previstos en la propia LDC". En segundo lugar, se establece, en el punto tercero, que con este texto la CNC "pretende establecer unas directrices que, con carácter general, guíen su actuación", señalando, lo cual nos parece sumamente relevante, que "con ello se pretende contribuir a mejorar la transparencia y la objetividad en el cálculo de la sanción", así como "potenciar su efecto disuasorio y favorecer la seguridad jurídica de los operadores económicos". Así pues, se explicita una relación entre margen de apreciación en la imposición de sanciones, establecimiento de guías de actuación y principio de objetividad, con una referencia adicional que nos parece muy relevante a la transparencia. Sin duda, un reconocimiento de márgenes sincero que va unido a un

32 Baño León, José María, Potestades administrativas y garantías de las empresas en el Derecho español de la competencia, Mc Graw Hill, Madrid, 1996, p. 239. 
autocontrol que debe redundar en un ejercicio más eficaz y responsable de los poderes sancionadores.

Otros dos aspectos hay que destacar en la formulación del texto de esta comunicación. En primer lugar, se hace constar (apartado cuarto) que la metodología establecida para la cuantificación de las sanciones "será aplicada con carácter general”, sin perjuicio de una aplicación circunstancial de otros criterios si es necesario; pero con la consiguiente necesaria motivación. Una relación entre aplicación general y excepcional que se articula, pues, con el deber de motivación y que también deberá repercutir en una mayor confianza y transparencia. En segundo lugar, se establece también (apartado séptimo) que a la vista de la experiencia acumulada durante la aplicación de esta comunicación se procederá a efectuar su revisión al transcurrir dos años desde su primera aplicación. Cuestión que conectaría con el carácter evolutivo y dinámico de algunos aspectos implicados en su actuación o simplemente con la conveniencia de reconsiderar ciertos aspectos técnicos.

Pero el interés de este texto no finaliza aquí. En su apartado 21 se lleva a cabo una operación que ejemplifica lo que en buena parte de este estudio se ha estado comentando: la tarea de concreción de un concepto de la ley. Así, se lleva a cabo la "delimitación del concepto de mercado de reciente liberalización que aparece en la tipificación de una infracción muy grave por abuso de posición dominante en el art. 62.4.b LDC. Y así, se entenderán por tales mercados los que están "en proceso de liberalización supervisados por autoridades regulatorias o mercados objeto de un proceso normativo tendente a favorecer la entrada de nuevos operadores".

Asimismo se ha publicado la Comunicación sobre terminación convencional de expedientes sancionadores, de octubre de 2011, en la cual también se establecen unas directrices generales que deberán mejorar la transparencia, la previsibilidad y la seguridad jurídica al respecto. Entre los criterios que se establecen, se indica, en el punto 17, que cada expediente sancionador presenta especificidades, de modo que la CNC "debe aplicar caso a caso el margen de apreciación del que dispone", partiendo de que las decisiones de inicio y aceptación tienen carácter discrecional para la CNC; señalándose también que los criterios de actuación se establecen por norma general (así, en los puntos 22 y 23).

En cuanto al segundo ámbito señalado, el del mercado de valores, también es un sector de considerable y creciente complejidad que obliga a tener muy en cuenta la necesidad de criterios en la aplicación de la normativa reguladora. Ello lo podemos comprobar en el Informe anual sobre los mercados de valores y su actuación de la Comisión Nacional del Mercado de Valores (CNMV) de 2010, donde se pueden ver "criterios de interés" por ejemplo en materia de comunicación de operaciones sospechosas de abuso de mercado ${ }^{33}$. También se pone de relieve, por ejemplo, la dificultad de

33 CNMV, Informe anual..., op. cit., p. 202. 
definir con "criterios objetivos" las informaciones que pueden tener una influencia sensible en la cotización de los valores y así ser consideradas relevantes, haciéndose referencia al contenido de una Orden Ministerial de 2009 en materia de información relevante ${ }^{34}$. Por citar un ejemplo relativo a este tipo de ámbitos de actuación del Derecho comparado, en el caso de la Autorité des Marchés Financiers francesa también se puede comprobar en su página web ${ }^{35}$ la atención dedicada a precisar criterios relativos por ejemplo a la concurrencia de una información privilegiada a la vista de los cuales la Comisión de sanciones de dicha autoridad puede establecer los incumplimientos. Es interesante indicar que se encuentran también ejemplos reales que muestran los factores que se tienen en cuenta para modular las sanciones, lo cual es un aspecto que puede ser destacable desde el punto de vista de la coherencia y la transparencia de la actuación sancionadora en este ámbito ${ }^{36}$.

Por otra parte, las recientes reformas en la legislación del mercado financiero en España, que persiguen incrementar ciertos mecanismos de control a la vista de la gravedad de ciertos comportamientos en el origen de la crisis desencadenada en 2008, están presentes cláusulas de carácter general que también es plausible pensar que exigirán el establecimiento de criterios concretos de aplicación. Así, por no poner más que algunos ejemplos, podemos observar algunas reformas introducidas por la Ley 2/2011, de 4 de marzo, de Economía Sostenible. Con carácter general su art. 28, mejora de la supervisión financiera, establece que "las autoridades financieras incorporarán al ejercicio de sus facultades de inspección y sanción los principios, normas y criterios de calidad incorporados en las disposiciones finales $5 .^{\mathrm{a}}, 7 .^{\mathrm{a}}, 8 .^{\mathrm{a}}, 9 .^{\mathrm{a}}$ y $10 .^{\mathrm{a}}$ de esta Ley", lo cual requerirá un notable esfuerzo de aplicación dada la gran amplitud de dichas disposiciones. Justamente como contenido de algunas de esas disposiciones finales citadas se realizan reformas de la Ley del Mercado de Valores que introducen conceptos amplios que requerirán criterios de concreción. Así, por ejemplo, en el art. 106 ter, que se añade, al establecerse criterios de determinación de las sanciones, se incluyen factores como "las consecuencias desfavorables de los hechos para el Sistema Financiero o la Economía Nacional” (apartado 1.e). O en el art. 107 bis, que también se añade, al regular la posible actuación de requerimiento sin carácter sancionador en casos de infracciones leves, estableciendo la necesidad de motivar en estos casos "la no afectación de la conducta de manera significativa a los intereses públicos protegidos por esta ley”.

En cualquier caso se pone de manifiesto la importancia de contar con criterios objetivos en la determinación de los distintos elementos de la resolución sancionadora. La aportación de instrumentos generales que predeterminen esos criterios es muy relevante, como hemos visto en el primer ejemplo. En otros ámbitos ello se ha establecido explícitamente a través de norma reglamentaria, como el caso reciente

34 CNMV, Informe anual..., op. cit., p. 203.

35 En el documento Les manquements relevant de la compétence de l'AMF, actualizado el 28 febrero 2011, pp. 2-3.

36 En el documento Le montant de la sanction pécuniaire, actualizado el 27 enero 2011, p. 5. 
del Real Decreto 1677/2011, de 18 de noviembre, en materia de función estadística pública, mediante el cual "se introducen criterios de calificación de las infracciones y de graduación de las sanciones en el ámbito del Departamento de Aduanas e Impuestos especiales de la Agencia Estatal de Administración Tributaria", de notable precisión.

La adecuada combinación entre los instrumentos generales de fijación de criterios, de diversa tipología, y la actuación singular en cada caso de la Administración, visualizada especialmente a través de la motivación de sus resoluciones, así como la adecuada combinación entre elementos de determinación externa de criterios y de autolimitación, resultarán aspectos sumamente relevantes para el respeto de las exigencias del principio de objetividad en materia sancionadora.

\section{CONSIDERACIÓN FINAL}

El rigor y la coherencia en la aplicación de los poderes de sanción serán importantes en el marco de una creciente preocupación por la necesaria firmeza, eficacia y efectividad del ejercicio de la potestad administrativa sancionadora, como ocurre en el sector de los servicios financieros, tal como ha puesto de relieve ya de modo muy explícito la Comisión de la Unión Europea37. Sancionar mejor, en la siempre difícil ponderación entre garantías y eficacia, puede resultar, en este sentido, especialmente importante para velar por los intereses generales en entornos complejos. Y sancionar mejor implica, sin ocultar ni cerrar en falso los espacios abiertos por los márgenes de apreciación existentes, objetivar mejor la actuación sancionadora. También en este ámbito, una Administración más transparente, coherente y objetiva, es una Administración más fuerte. La presentación aquí llevada a cabo sobre las líneas a explorar en la aplicación del principio de objetividad en el ejercicio de la potestad sancionadora intenta ponerlo de relieve.

\section{BIBLIOGRAFÍA CITADA}

Bacigalupo, Mariano, La discrecionalidad administrativa (estructura normativa, control judicial y límites constitucionales de su atribución), Marcial Pons, Madrid, 1997.

Baño León, José María, Potestades administrativas y garantías de las empresas en el Derecho español de la competencia, Mc Graw Hill, Madrid, 1996.

Huergo Lora, Alejandro, Las sanciones administrativas, lustel, Madrid, 2007.

Igartua Salaverría, Juan, Discrecionalidad técnica, motivación y control jurisdiccional, Civitas, Madrid, 1998.

37 Concretamente en una muy destacable Comunicación, de 8 diciembre 2010, al Parlamento Europeo, al Consejo, al Comité Económico y Social Europeo y al Comité de las Regiones, titulada Regímenes sancionadores más rigurosos en el sector de servicios financieros. 
Igartua Salaverría, Juan, La motivación en los nombramientos discrecionales, Thomson-Civitas, Cizur Menor, 2007.

Izquierdo Carrasco, Manuel, “La determinación de la sanción administrativa”, en Justicia Administrativa, número extraordinario, 2001.

Izquierdo Carrasco, Manuel, "La resolución sancionadora”, en Rebollo Puig, Manuel; Izquierdo Carrasco, Manuel; Alarcón Sotomayor, Lucía y Bueno Armijo, Antonio M. ${ }^{a}$, Derecho Administrativo Sancionador, Lex Nova, Valladolid, 2010.

Lasagabaster Herrarte, Iñaki, “Artículo 4. Tipicidad”, en Lasagabaster Herrarte, Iñaki (dir.), Ley de la potestad sancionadora. Comentario sistemático, LETE, IVAP, Bilbao - Iruña, 2006.

Malaret i García, Elisenda, “Autoridades independientes y justicia administrativa”, en Montoro Chiner, María Jesús (coord.), La Justicia Administrativa. Libro homenaje al Prof. Dr. D. Rafael Entrena Cuesta, Atelier, Barcelona, 2003.

Morell Ocaña, Luis, "El principio de objetividad en la actuación de la Administración Pública”, en Martín-Retortillo Baquer, Lorenzo (coord.), La protección jurídica del ciudadano (procedimiento administrativo y garantía jurisdiccional): estudios en homenaje al profesor Jesús González Pérez, Civitas, Madrid, 1993.

Nieto García, Alejandro, "La Administración sirve con objetividad los intereses generales”, en Martín-Retortillo Baquer, Sebastián (coord.), Estudios sobre la Constitución española. Homenaje al profesor Eduardo García de Enterría, Civitas, Madrid, 1991.

Nieto García, Alejandro, Derecho Administrativo Sancionador, Tecnos, Madrid, $4 .^{\text {a }}$ ed., 2005.

Pemán Gavín, Ignacio, El sistema sancionador español. Hacia una teoría general de las infracciones y sanciones administrativas, Cedecs, Barcelona, 2000.

Ponce Solé, Juli, Deber de buena administración y derecho al procedimiento administrativo debido. Las bases constitucionales del procedimiento administrativo y del ejercicio de la discrecionalidad, Lex Nova, Valladolid, 2001.

Sánchez Morón, Miguel, Derecho Administrativo. Parte general, Tecnos, Madrid, $7 .^{\text {a }}$ ed., 2011.

Santamaría Pastor, Juan Alfonso, Principios de Derecho Administrativo General I, lustel, Madrid, $2 .^{\text {a }}$ ed., 2009.

Scoletta, Marco, “El principio de legalidad penal europeo”, en Díez-Picazo, Luis María y Nieto Martín, Adán (dirs.), Los derechos fundamentales en el Derecho Penal Europeo, Civitas-Thomson, Cizur Menor, 2010.

Simon, François-Luc, Le juge et les autorités du marché boursier, LGDJ, París, 2004. 
